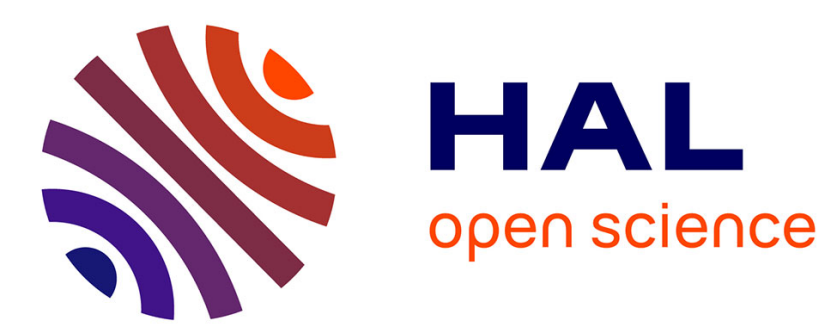

\title{
A semi-analytical finite element method for elastic guided waves propagating in helical structures
}

Fabien Treyssede, Ahmed Frikha

\section{To cite this version:}

Fabien Treyssede, Ahmed Frikha. A semi-analytical finite element method for elastic guided waves propagating in helical structures. Acoustics' 08, Jun 2008, France. 5p. hal-00639665

\section{HAL Id: hal-00639665 \\ https://hal.science/hal-00639665}

Submitted on 9 Nov 2011

HAL is a multi-disciplinary open access archive for the deposit and dissemination of scientific research documents, whether they are published or not. The documents may come from teaching and research institutions in France or abroad, or from public or private research centers.
L'archive ouverte pluridisciplinaire HAL, est destinée au dépôt et à la diffusion de documents scientifiques de niveau recherche, publiés ou non, émanant des établissements d'enseignement et de recherche français ou étrangers, des laboratoires publics ou privés. 

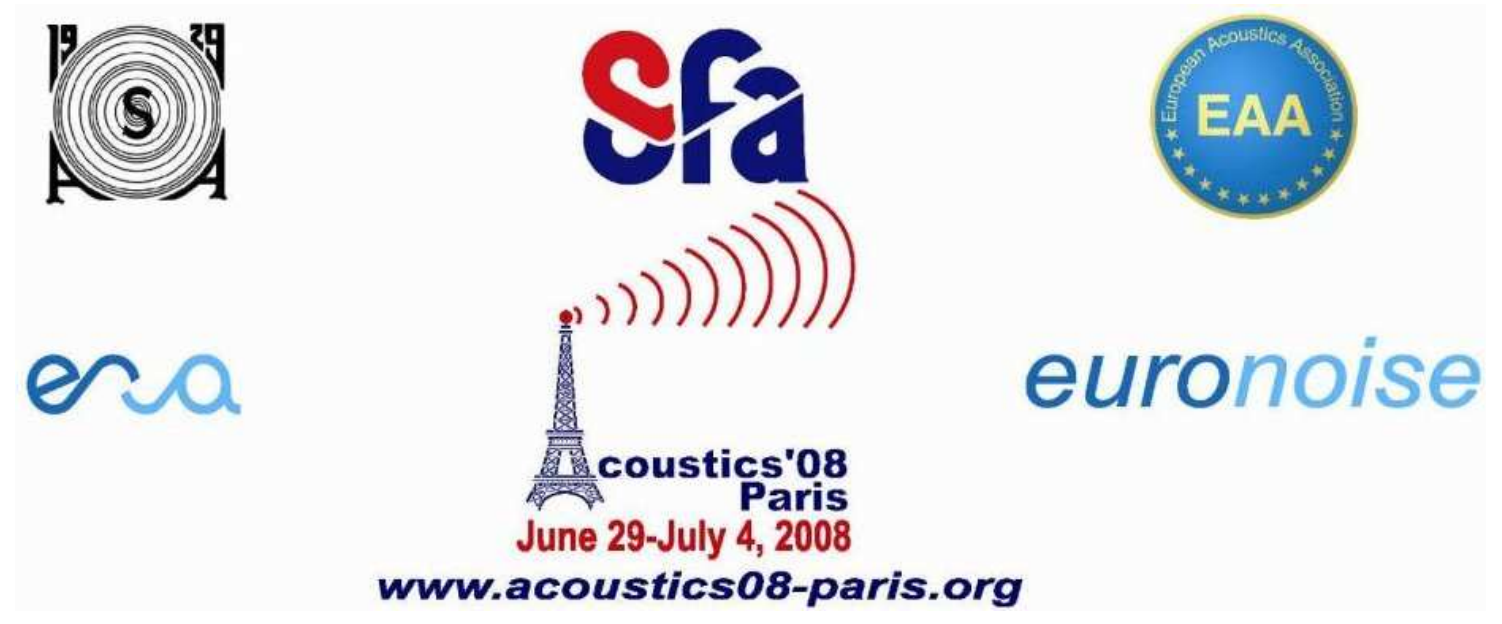

A semi-analytical finite element method for elastic guided waves propagating in helical structures

F. Treyssède and A. Frikha

Nantes Atlantique University - LCPC, Division for Metrology and Instrumentation, Route de Pornic - BP 4129, 44341 Bouguenais, France

fabien.treyssede@lcpc.fr 
Steel multi-wire cables are widely used in civil engineering as load-carrying members. The basic element of these cables is usually a simple straight strand made of a straight core and one layer of helical wires. Several difficulties arise in the understanding of guided ultrasonic waves in such structures, partly due to the helical geometry and the inter-wire coupling effects. In the context of non-destructive evaluation, this work aims at theoretically investigating the propagation of elastic waves in helical waveguides. A numerical method is chosen based on a semi-analytical finite element technique that relies on a specific non-orthogonal curvilinear coordinate system. This system is shown to be translationally invariant along the helix centerline so that a spatial Fourier transform can be explicitly performed along the axis and the problem is reduced to two dimensions. It is shown that the method can be readily used for the analysis of helical structures by considering the special case of no curvature. Results for single straight and helical wires are first computed in order to validate the approach. A dispersion analysis for a seven wire strand with simplified contact conditions is then realized.

\section{Introduction}

In civil engineering, steel mutli-wire cables are widely employed. The basic element of these cables is usually a simple straight strand made of a straight core and one layer of helical wires. Mainly because of corrosion, material degradation of steel may result in fractures of wires. A structural health monitoring approach based on nondestructive evaluation methods is strongly needed in order to prevent such failures. Ultrasonics is one of the most popular techniques. It consists in analyzing the propagation of elastic guided waves, which are known to be multimodal and dispersive. For a better physical understanding of these complex effects, some theoretical models are often required.

Some recent experimental studies of multi-wire strands have been realized by Kwun et al. [1], Beard et al. [2] and Rizzo et al. [3] pointing out the fact that the cylindrical model approximation cannot accurately predict propagation inside multi-wire strands. In fact, the theoretical understanding of guided ultrasonic waves in multi-wire strands is complicated by the helical geometry of peripheral wires, the inter-wire coupling and contact effects, and the presence of applied loads.

The goal of this paper is to address the first above complicating effect, by proposing a numerical method allowing the study of elastic guided waves inside a single helical wire. It is shown that the proposed method can also be readily used to study some general helical structures, having both straight and helical wires. Inter-wire coupling effects are included in the analysis through simplified contact conditions.

In order to deal with complex geometry, some of the most popular and efficient numerical techniques are based on finite element (FE) methods. The so-called semi-analytical finite element (SAFE) method is a first approach that has been used to study uniform waveguides of arbitrary crosssection - see for instance, Gavric [4], Hayashi et al. [5], Damljanovic et al. [6]. Demma et al. [7] investigated toroidal waveguides. Onipede et al. [8] extended SAFE methods to study uniformly pretwisted waveguides along a straight axis.

A second approach is based on the theory of wave propagation in periodic structures from Floquet's principle. A review on the topic can be found in Mead [9]. Based on a general theory presented by Mead [10], some periodic FE approaches and procedures have then been developed - see for instance Gry et al. [11], Mace et al. [12].

For modelling a single helical wire, which is a uniform waveguide, both SAFE and periodic FE approaches can be applied. Treyssede [13] has recently proposed a numerical procedure based on a periodic FE approach combined with a specific helical mapping in order to arbitrarily reduce the periodic cell length. In this paper, a SAFE method extended to helical waveguides is developed. The weak variational formulation is written in terms of a non-orthogonal curvilinear coordinate system that is translationally invariant along the helix centreline, so that a Fourier transform can be explicitly performed. It is also shown how the formulation can be readily used in order to analyse multi-wire strands. As opposed to a periodic FE approach, this approach explicitly takes into account the property of translational invariance of helical waveguides. More mathematical insight is somewhat gained and the problem to be solved is reduced to two dimensions (hereby reducing computational costs).

\section{SAFE method}

A linearly elastic material, small strains and displacements and a time harmonic dependence are assumed. There is no external force. The 3D variational formulation governing elastodynamics is then given by:

$$
\int_{\Omega} \delta \boldsymbol{\epsilon}^{T} \mathbf{C} \boldsymbol{\epsilon} d V-\omega^{2} \int_{\Omega} \rho \delta \mathbf{u}^{T} \mathbf{u} d V
$$

for any kinematically admissible trial displacement field $\delta \mathbf{u} . \quad \delta \boldsymbol{\epsilon}$ denotes the virtual strain vector $\left[\begin{array}{lllllll}\delta \epsilon_{11} & \delta \epsilon_{22} & \delta \epsilon_{33} & 2 \delta \epsilon_{12} & 2 \delta \epsilon_{13} & 2 \delta \epsilon_{23}\end{array}\right]^{T}$. Subscripts $i=1,2,3$ are components in the considered coordinate system, respectively denoted $(x, y, s)$. C is the matrix of material properties. $\rho$ is the material density and $\Omega$ is the structural volume. In the remainder of this paper, $s$ will denote the waveguide axis.

The strain-displacement relation can be written as follows:

$$
\boldsymbol{\epsilon}=\left(\mathbf{L}_{x y}+\mathbf{L}_{s} \partial / \partial s\right) \mathbf{u}
$$

where $\mathbf{L}_{x y}$ is the operator containing all terms that do not contain derivatives with respect to the axis $s$. Now we further assume a dependence $e^{i k s}, k$ being the axial wavenumber. The problem is hence reduced from three to two dimensions (from the volume $\Omega$ to the cross-section $S$ of the waveguide). Then, it can be shown that the FE discretisation of Eq.(1) finally gives the following eigenvalue problem:

$$
\left\{\mathbf{K}_{1}-\omega^{2} \mathbf{M}+i k\left(\mathbf{K}_{2}-\mathbf{K}_{2}^{T}\right)+k^{2} \mathbf{K}_{3}\right\} \mathbf{u}=\mathbf{0}
$$

with the following elementary matrices: 


$$
\begin{gathered}
\mathbf{K}_{1}^{e}=\int_{S^{e}} \mathbf{N}^{e T} \mathbf{L}_{x y}^{T} \mathbf{C} \mathbf{L}_{x y} \mathbf{N}^{e} d S, \quad \mathbf{K}_{2}^{e}=\int_{S^{e}} \mathbf{N}^{e T} \mathbf{L}_{x y}^{T} \mathbf{C} \mathbf{L}_{s} \mathbf{N}^{e} d S \\
\mathbf{K}_{3}^{e}=\int_{S^{e}} \mathbf{N}^{e T} \mathbf{L}_{s}^{T} \mathbf{C} \mathbf{L}_{s} \mathbf{N}^{e} d S, \mathbf{M}^{e}=\int_{S^{e}} \rho \mathbf{N}^{e T} \mathbf{N}^{e} d S
\end{gathered}
$$

The solution of Eq. (3) yields the propagation modes. At fixed real $k$, the eigenproblem (3) is linear for finding $\omega^{2}$. This simpler approach is useful only if interest is restricted to propagating modes in undamped systems. Given $\omega$ and finding $k$, the eigenproblem is quadratic. It can be recast into a generalized linear eigensystem written for $\left[\begin{array}{ll}\mathbf{u} & k \mathbf{u}\end{array}\right]^{T}$ in order to be solved by standard numerical solvers - see Tisseur et al. [14] for instance.

\section{Translationally invariant helical coordinate system}

In the previous section, we have assumed an $e^{i k s}$ dependence. This is equivalent to perform a spatial Fourier analysis in the $s$ direction. This assumption indeed requires that the physical system be translationally invariant. In other words, this means that the cross-section of the waveguide must not vary along $s$ (condition 1), nor the material properties (condition 2, which is assumed to be satisfied throughout this work). Besides, the $(x, y, s)$ coordinate system must be so that $s$ do not appear explicitly in the coefficients of the equilibrium equations ( $s$ must only appear through derivatives). Verifying this last condition (condition 3) is somewhat more technical than the first two.

First, let us define the helix centerline curve. It can be described by the following position vector in the Cartesian orthonormal basis:

$$
\mathbf{R}(s)=R \cos \left(\frac{2 \pi}{l} s\right) \mathbf{e}_{X}+R \sin \left(\frac{2 \pi}{l} s\right) \mathbf{e}_{Y}+\frac{L}{l} s \mathbf{e}_{Z}
$$

where $l=\sqrt{\left(L^{2}+4 \pi^{2} R^{2}\right)} . R$ and $L$ are respectively the radius of the centreline in the $(X, Y)$ Cartesian plane and the helix step along the $Z$ axis. The unit tangent, normal and binormal vectors to the centreline are respectively obtained from $\mathbf{T}=d \mathbf{R} / d s$ and the Serret-Frenet formulae, $d \mathbf{T} / d s=\kappa \mathbf{N}$ and $d \mathbf{N} / d s=\tau \mathbf{B}-\kappa \mathbf{T}$. Both the curvature $\kappa=4 \pi^{2} R / l^{2}$ and the tortuosity $\tau=2 \pi L / l^{2}$ are constant.

Now, a new coordinate system is constructed from the orthonormal basis $(\mathbf{N}, \mathbf{B}, \mathbf{T})$ as follows:

$$
\mathbf{X}(x, y, s)=\mathbf{R}(s)+x \mathbf{N}(s)+y \mathbf{B}(s)
$$

yielding the covariant basis $\left(\mathbf{g}_{1}, \mathbf{g}_{2}, \mathbf{g}_{3}\right)=(\partial \mathbf{X} / \partial x, \partial \mathbf{X} / \partial y, \partial \mathbf{X} / \partial s)$ (non-orthogonal). It could be checked that the metric tensor, defined by $(\mathbf{g})_{i j}=\mathbf{g}_{i} \cdot \mathbf{g}_{j}$, does not depend on the third curvilinear coordinate $s$. One consequence is that the coefficients of the partial differential equilibrium equations are not dependent upon $s$ either (condition 3 is satisfied). For clarity, $\mathbf{L}_{x y}$ and $\mathbf{L}_{s}$ are explicitly given by:

$$
\mathbf{L}_{x y}=\left[\begin{array}{ccc}
\partial / \partial x & 0 & 0 \\
0 & \partial / \partial y & 0 \\
\tau^{2} x-\kappa(1-\kappa x) & \tau^{2} y & -\kappa \tau y \\
\partial / \partial y & \partial / \partial x & 0 \\
-\tau y \partial / \partial x & -\tau+\tau x \partial / \partial x & \kappa+(1-\kappa x) \partial / \partial x \\
\tau-\tau y \partial / \partial y & \tau x \partial / \partial y & (1-\kappa x) \partial / \partial y
\end{array}\right]
$$

and:

$$
\mathbf{L}_{s}=\left[\begin{array}{ccc}
0 & 0 & 0 \\
0 & 0 & 0 \\
-\tau y & \tau x & 1-\kappa x \\
0 & 0 & 0 \\
1 & 0 & 0 \\
0 & 1 & 0
\end{array}\right]
$$

For Eqs.(3)-(4), (7) and (8) to hold, it must be outlined that covariant components have been chosen for $\boldsymbol{\epsilon}$ while components with respect to $(\mathbf{N}, \mathbf{B}, \mathbf{T})$ have been preferred for $\mathbf{u}$. Also, the components of $\mathbf{C}$ should be understood as contravariant - for more details, see Treyssède [15].

Now, let us consider a single helical wire of circular crosssection. The cross-section obviously does not change along the helical axis $s$ (condition 1 is fulfilled) so that the proposed approach is valid. The analysis of a straight wire can also be readily performed, the cylinder corresponding to the special case $\kappa=\tau=0$.

However, a question arises about the choice of the invariant coordinate system to be used when considering helical structures made of both straight and helical wires. Of course, the choice $\kappa=\tau=0$ (resp. $\kappa \neq 0, \tau \neq 0$ ) cannot be applied because condition 1 would not be satisfied for helical (resp. straight) wires.

The adequate system is indeed given by $\kappa=0 \quad(R=0)$, $\tau=2 \pi / L$, corresponding to a rotating coordinate system along the $Z$ axis $(s \equiv Z)$. With this system, a central straight wire (cylinder) has an invariant circular crosssection along $Z$ ("a twisted cylinder remains a cylinder"). Furthermore, the cross-sections of peripheral helical wires do not change either along $Z$, although their shape is not circular any more in the $(x, y)$ plane. These statements will be verified in Section 4.1. Note that such a rotating system coincides with the one proposed by Onipede et al. [8] for the analysis of twisted waveguides.

\section{Numerical results}

The material is assumed to be isotropic, with no material damping. For a steel wire, a typical value of 0.30 will be chosen for the Poisson coefficient. We consider waveguides with a circular cross-section of radius $a$. The adimensionalized angular frequency is given by $\omega a / c_{s}, c_{s}$ denoting the shear velocity. Six-node triangles meshes will be used. FE computations are held at fixed real wavenumbers $k$.

\subsection{Dispersion analysis of single wires}

A single straight wire (cylinder) is first considered $(\kappa=0)$. Figure 1a exhibits the cross-section FE mesh used. Both 
cases $\tau=0$ (reference solution) and $\tau \neq 0$ are computed. In order to check the adequacy of the rotating system, we choose a high tortuosity $\tau a=0.5$ (high rotating rate). Figure 2 exhibits the dispersion curves for both cases. Though strongly different, one must be careful when interpreting results: wavenumbers of flexural modes $F(m, n)$ are modulated by $\pm m \alpha a$ because of the system rotation, while those of compression and torsional modes are left unchanged - see Onipede et al. [8] for further explanations. Hence, both results yield the same physical phenomenon. This is clearly outlined by Fig. 3 giving the energy velocity vs. frequency (same curves obtained for both straight and rotating systems).
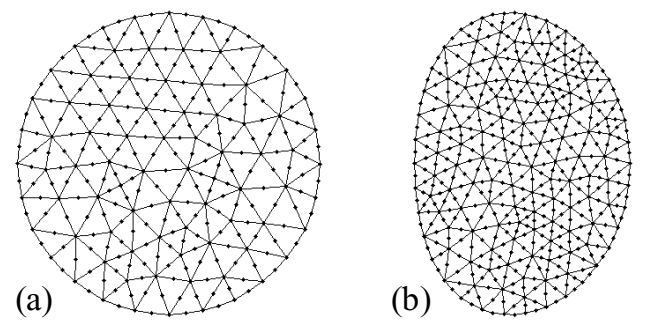

Fig. 1 Cross-section FE meshes in the $(X, Y)$ plane for: (a) the cylinder, (b) the helical waveguide $\phi=45^{\circ}$ when a rotating system $\kappa=0$ is used (cross-section centred at $(X, Y)=(2 a, 0))$.

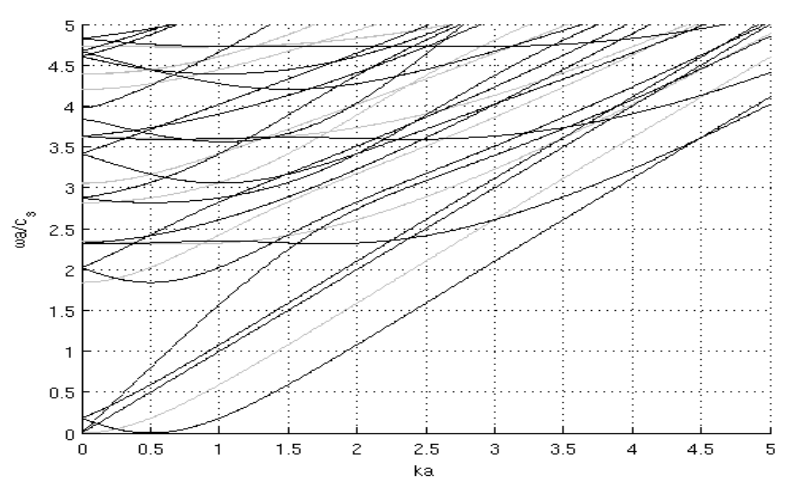

Fig.2 Cylindrical waveguide. Plot of frequency vs. wavenumber. Black lines: rotating system, gray lines: Cartesian system.

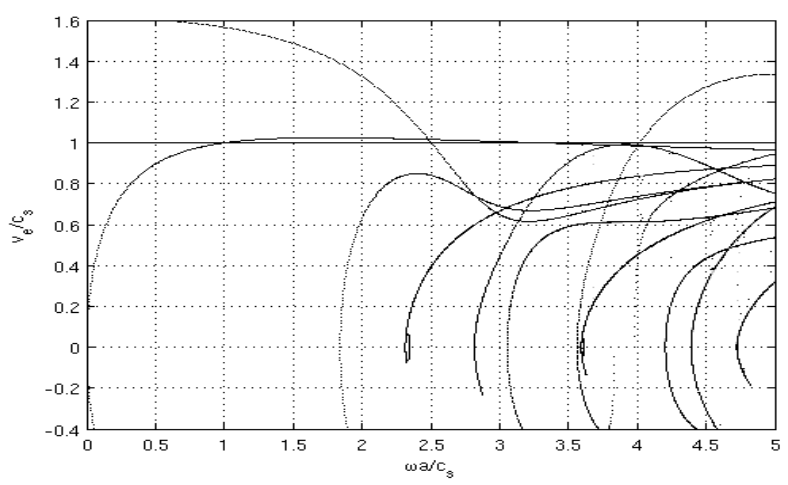

Fig.3 Cylindrical waveguide. Plot of energy velocity vs. frequency. Black lines: rotating system, gray lines: Cartesian system.
Now, a single helical wire is considered. The helix radius is $R=2 a$. A strong helix lay angle $\phi=\tan ^{-1}(2 \pi R / L)=\pi / 4 \quad$ is chosen, yielding ( $\kappa a=0.25, \tau a=0.25)$ for the helical system and ( $\kappa a=0, \tau a=0.5$ ) for the rotating system. The computed solution given by the helical system, for which the crosssection is circular (same as Fig. 1a), is considered as the reference solution (see Refs. [13, 15]). Fig 1b exhibits the cross-section mesh that must be used together with the rotating system $((X, Y)$ plane cut of the helical waveguide). Figure 4 exhibits the dispersion curves computed for both coordinate systems. Here again, one must be careful when comparing results: in order to transform results from the curvilinear $s$ axis to the Cartesian $Z$ axis, wavenumbers $k$ obtained from the helical system must be multiplied by the ratio $l / L$. As can be observed in Fig 4, no difference are found between both systems, which demonstrates the adequacy of the rotating system. Note that the differences previously observed for the cylinder between flexural modes do not occur here because both coordinates systems rotate with the same rate along the $Z$ axis.

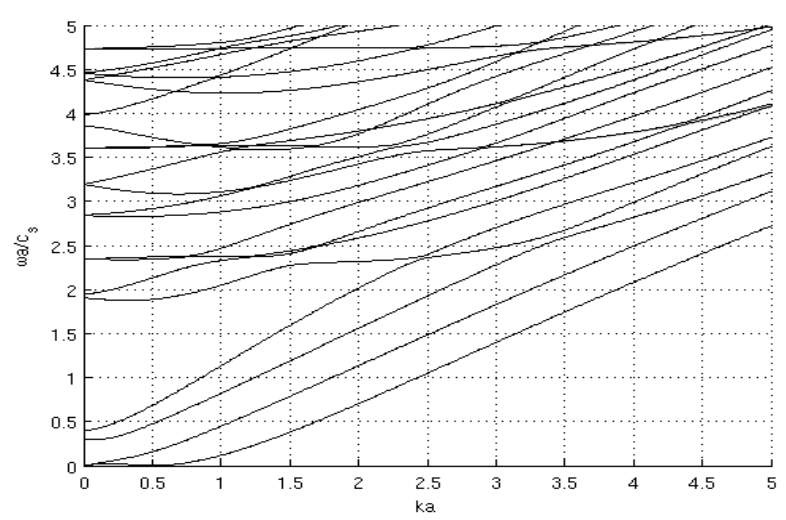

Fig.4 Helical waveguide ( $\phi=45^{\circ}$ ). Plot of frequency vs. wavenumber. Black lines: rotating system, gray lines: helical system.

\subsection{Dispersion analysis of a seven-wire strand}

The wave modes propagating inside a typical seven-wire strand are now studied. Stick contact conditions are assumed for simplicity (no slip, no separation and no friction are considered). $a$ denotes the central wire radius $(a=2.5 \mathrm{~mm})$. Peripheral wires radii are chosen as $0.97 a$, with a lay angle $\phi=7.5^{\circ}$ (typical for civil engineering $6+1$ strands). The coordinate system to be used is $(\kappa a=0, \tau a=0.0668)$. The FE mesh is given in Fig. 5 . Figures 6 and 7 exhibit the dispersion curves for the adimensional frequency range [0;2]. Due to a strong interwire coupling, one observes a far more complex behaviour than for single wires. The most striking phenomenon is the cut-off of the fastest mode (compressional-like $L(0,1)$ mode) around $\omega a / c_{s}=0.6$, corresponding to $120 \mathrm{kHz}$ as experimentally observed by Kwun et al. [1]. 


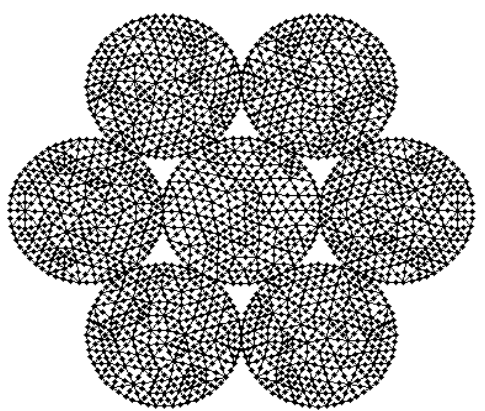

Fig.5 Cross-section FE mesh in the $(X, Y)$ plane for the seven-wire strand $\left(\phi=7.5^{\circ}\right)$.

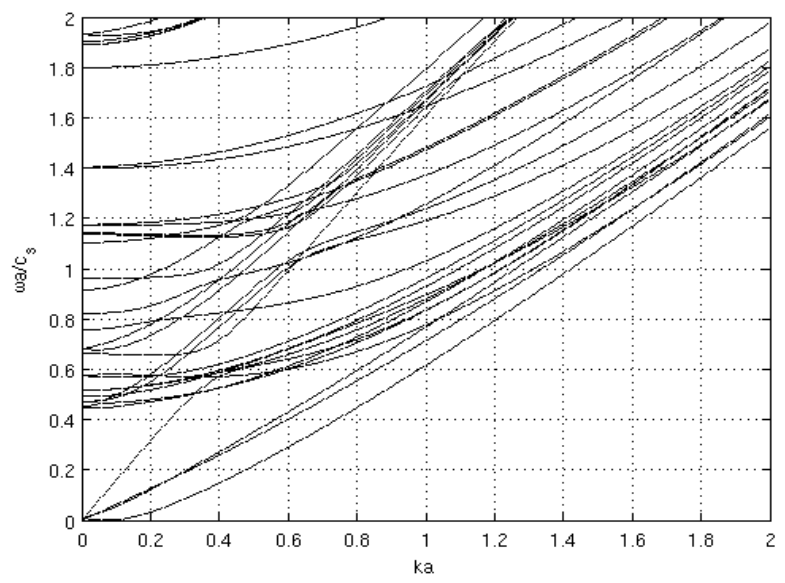

Fig.6 Seven-wire strand $\left(\phi=7.5^{\circ}\right)$. Plot of frequency vs. wavenumber.

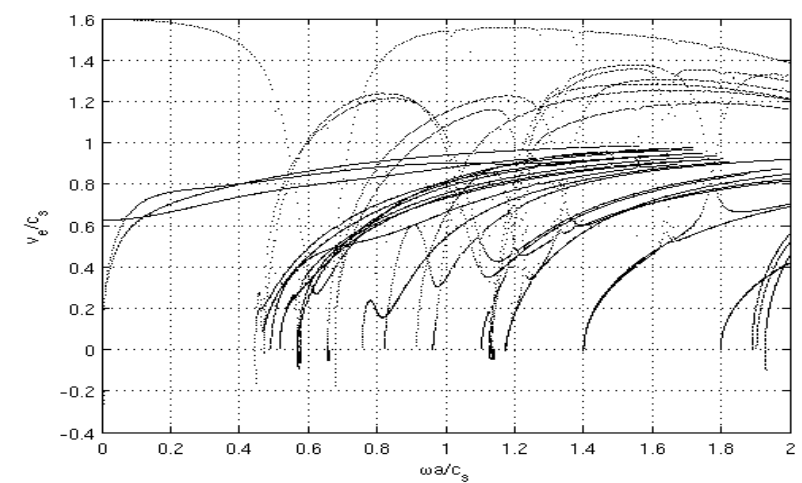

Fig.7 Seven-wire strand ( $\left.\phi=7.5^{\circ}\right)$. Plot of energy velocity vs. frequency.

\section{Conclusion}

Elastic wave propagation inside helical wires has been analysed through a SAFE method based on a translationally invariant helical coordinate system. It has been shown that the special case of no curvature yields an adequate coordinate system to be used for the analysis of helical structures made of a straight core and peripheral helical wires. Dispersion inside a typical $6+1$ strand has then been investigated by assuming stick contact conditions. A far more complex behaviour than for single wires has been observed due to a strong inter-wire coupling. Besides, a bandcut zone is found for the fastest compressional-like mode, centred near $120 \mathrm{kHz}$, which seems to coincide with experimental results mentioned in the literature.

\section{References}

[1] H. Kwun, K. A. Bartels, J. J. Hanley, "Effects of tensile loading on the properties of elastic-wave propagation in a strand", Journal of the Acoustical Society of America 103, 3370-3375 (1998)

[2] M. D. Beard, M. J. S. Lowe, P. Cawley, "Ultrasonic guided waves for inspection of grouted tendons and bolts", Journal of Materials in Civil Engineering 212, 212-218 (2003)

[3] P. Rizzo, F. Lanza di Scalea, "Wave propagation in multi-wire strands by wavelet-based laser ultrasound", Experimental Mechanics 44, 407-415 (2004)

[4] L. Gavric, "Computation of propagative waves in free rail using a finite element technique", Journal of Sound and Vibration 185, 531-543 (1995)

[5] T. Hayashi, W.-J. Song, J. L. Rose, "Guided wave dispersion curves for a bar with an arbitrary cross-section, a rod and rail example", Ultrasonics 41, 175-183 (2003)

[6] V. Damljanovic, R. L. Weaver, "Propagating and evanescent elastic waves in cylindrical waveguides of arbitrary cross-section", Journal of the Acoustical Society of America 115, 1572-1581 (2004)

[7] A. Demma, P. Cawley, M. Lowe, "The effect of bends on the propagation of guided waves in pipes", Journal of Pressure Vessel Technology 127, 328-335 (2005)

[8] O. Onipede, S. B. Dong, "Propagating waves and end modes in pretwisted beams", Journal of Sound and Vibration 195, 313-330 (1996)

[9] D. J. Mead, "Wave propagation in continuous periodic structures: research contributions from Southampton, 1964-1995", Journal of Sound and Vibration 190, 495-524 (1996)

[10] D. J. Mead, "A general theory of harmonic wave propagation in linear periodic systems with multiple coupling", Journal of Sound and Vibration 27, 235-260 (1973)

[11] L. Gry, C. Gontier, "Dynamic modeling of railway track: a periodic model based on a generalized beam formulation", Journal of Sound and Vibration 199, 531558 (1997)

[12] B. R. Mace, D. Duhamel, M. J. Brennan, "Finite element prediction of wave motion in structural waveguides", Journal of the Acoustical Society of America 117, 2835-2843 (2005)

[13] F. Treyssede, "Numerical investigation of elastic modes of propagation in helical waveguides", Journal of the Acoustical Society of America 121, 3398-3408 (2007)

[14] F. Tisseur, K. Meerbergen, "The Quadratic Eigenvalue Problem", SIAM Review 43, 235-286 (2001)

[15] F. Treyssede, "Elastic waves in helical waveguides", Wave Motion, in press (2008) 